\title{
Lens Status
}

National Cancer Institute

\section{Source}

National Cancer Institute. Lens Status. NCI Thesaurus. Code C120718.

The condition or state of the lens of the eye. 